\title{
Evaluating performance of the Revised Trauma Score as a triage instrument in the prehospital setting
}

\author{
J. Roorda ${ }^{1}$, E. F. van Beeck ${ }^{2}$, J. W. J. L. Stapert ${ }^{3}$ and W. ten Wolde ${ }^{1}$ \\ ${ }^{1}$ Community Health Service 'Regio Twente', Enschede, 'Department of Public Health, Erasmus University Medical \\ School, Rotterdam, and 'Department of Surgery, Hospital 'Medisch Spectrum Twente', Enschede, The Netherlands
}

\begin{abstract}
In this study, we have evaluated the performance of the Revised Trauma Score (RTS) as a triage instrument in the prehospital setting in The Netherlands. To this end we analysed prehospital and clinical data on 398 injured patients in an urban-rural area in the east of the Netherlands. Our study included injured patients aged over 15 who were alive at the time the ambulance arrived. We found a comparatively low prevalence of major injuries in the prehospital setting, which varied with the definition used (for patients with an HTI-ISS $\geqslant 18$, it was 5.8 per cent, for HTI-ISS $\geqslant 20$ it was 3.7 per cent; for a modified HTI-ISS criterion it was 5.3 per cent and 2.7 per cent needed major emergency therapy). Estimates of sensitivity were also rather low and varied with the definition used (38 per cent for HTI-ISS $\geqslant 18 ; 56$ per cent for HTI-ISS $\geqslant 20,45$ per cent for the modified HTI-ISS criterion and 76 per cent for major emergency treatment). The specificity and the predictive value of a lowered RTS, however, were 94 per cent and 26 per cent respectively for all definitions used. The conclusion of this study is that the performance of the RTS in this study population is poorer than expected from earlier studies. The low prevalence of major injuries in the prehospital setting in The Netherlands and the distribution of case severity may possibly explain these results. Copyright (C) 1996 Elsevier Science Ltd. All rights reserved.
\end{abstract}

Injury, Vol. 27, No. 3, 163-167, 1996

\section{Introduction}

Improvement of the quality of medical care for injured patients is a topical issue in The Netherlands. This discussion was initiated in the early 1980 s because foreign studies provided evidence for substantially lower preventable death rates in regions with more advanced Trauma Care systems ${ }^{1}$. Since then a limited number of relevant studies have been carried out in The Netherlands as well. One study found a significantly lower fatality rate in hospitals offering more advanced trauma care (e.g. neurosurgery $)^{2}$, while an analysis of regional mortality differences within The Netherlands showed a significant negative correlation between the availability of an advanced trauma hospital in the region and the mortality due to road traffic accidents ${ }^{3}$.

These research findings provide support for the assumption that improvements in trauma care could bring a further substantial decline in accident mortality in The
Netherlands. For this reason, the regionalization of trauma care facilities has been advised in this country ${ }^{4}$. This, however, necessarily involves an adequate selection system to ensure that injured patients are treated in the right facility. This means that efforts must be made to keep 'under-triage' (i.e. failure to treat severely injured patients in a highly advanced hospital) and 'over-triage' (i.e. treatment of minor casualties in a highly advanced hospital) to the minimum. In The Netherlands, these triage decisions must be made in the prehospital setting by ambulance attendants. Ambulances are increasingly staffed by paramedics trained to supply advanced life support. There is a national network of emergency assistance, which - in line with national standards - can be present within $15 \mathrm{~min}$ of an emergency call. The distances to hospitals are generally short. In The Netherlands, the Revised Trauma Score (RTS, triage edition) has been proposed as the standard instrument for assessing the severity of injuries in accident victims.

Information on the predictive values of the RTS that is valid for the prehospital setting is scarce. In the first validation study on an in-patient population, Champion et al. ${ }^{5}$ found that almost 60 per cent of severely injured patients could be recognized by the RTS (sensitivity) and that almost 70 per cent of the patients with a low RTS had major injury (predictive value of a positive test). A study performed by Gilpin et al. ${ }^{\circ}$ resulted in the recognition of 80 per cent of the severely injured patients, while almost 50 per cent of all patients with a lower RTS had major injuries. Only one study was performed in a prehospital setting ${ }^{7 / 8}$. This study found high diagnostic qualities ( 85 per cent recognition of severely injured patients) but the percentage of severely injured patients among the group with a lowered RTS was relatively low ( 40 per cent). This study concluded that the RTS did not discriminate better than the informal judgement of emergency medical technicians.

The present study was undertaken to evaluate the performance of RTS when used by ambulance attendants in The Netherlands, who have to take a triage decision whether to transport a patient to an advanced trauma hospital. More specifically an answer was sought to the following questions:

What is the prevalence of major injuries in accident victims who are transported to a hospital by ambulance? 
What is the relationship between the RTS stated by ambulance personnel and the existence of severe injuries as diagnosed in the hospital? In which way is this relationship influenced by the way severe injuries are defined?

Is there a modifying effect on the performance of the RTS of variables such as age, sex, the circumstances of the accident, and the type of injury?

\section{Materials and methods}

\section{Inclusion of patients}

This study was carried out within a region in the east of The Netherlands, where all the ambulance assistance is supplied by a single community health service. The area of the region is about $350 \mathrm{~km}^{2}$, and it has almost 220000 inhabitants. There are two urban communities (150000 and 30000 inhabitants), surrounded by a more rural area. It has one hospital with advanced trauma-facilities and one regional hospital without. More than 90 per cent of injured patients are transported to one of the two hospitals. The ambulances are all staffed by paramedics, trained to give advanced life support.

Accident victims are defined as patients with physical or physiological injury following any sudden event with an external cause, for which immediate ambulance assistance is needed. In 1992 ambulances were called for 1308 accident victims. For all these patients, the RTS was measured at the time the ambulance arrived at the scene. During transport the RTS is regularly re-estimated. Data were recorded for each patient on a precoded form by the ambulance personnel and entered in a computerized database of the Community Health Service. The study population was formed retrospectively by analysing data in this database.

Out of the 1308 accident victims, patients were admitted in the study population if they met the following criteria: over 15 years of age; having an accident between May and December 1992; the triage decision was made by the ambulance attendants; immediate transportation to the emergency room of one of the two hospitals in the region; they were not obviously dead when the ambulance arrived. No further restrictions were made in the severity or characteristics of the cases. There were 712 patients who met the selection criteria. In this group, 657 patients $(92$ per cent) had an RTS of 12 (the highest score), and a random sample of 343 was taken in the study population. All 55 patients with an RTS lower than 12 were also included in the study population. This made a total study population consisting of 398 patients.

\section{Estimating the severity of injuries}

It was essential to identify those patients who should have been referred to a regional trauma centre. The injury severity score (ISS) is a well accepted measure, but there is no consensus as to which trauma patients should be referred to advanced trauma centre facilities; in the literature, several criteria are used. Because of this lack of a well accepted 'golden standard' we decided to use four different definitions. Three definitions related to the severity of injuries and the fourth to the necessity of major emergency therapy. The ISS in this study was based on the hospital trauma index $(\mathrm{HTI})^{10,11}$. The HTI scores injuries in six different organ systems or body regions (abdomen, respiratory system, cardiovascular system, central nervous system, extremities, and the skin and subcutis). The injuries in each category are rated between 0 (no injury) and 5 (very severe injury). The ISS sums the squares of the three highest scores of the HTI and ranges between 0 and 75 . According to the first definition of our study, trauma centre candidates have an HTI-ISS of 18 or over, a criterion that is often used in the literature ${ }^{11}$. In our second definition, an HTI-ISS of 20 or over is used, which excludes most patients with one 'major' bone fracture. Our third definition (the modified HTI-ISS criterion) was derived from panel criteria developed in earlier studies; ; every patient with at least one single serious injury or two moderate injuries in different body regions or organ systems is defined as a candidate for evaluation at a trauma centre. This means at least one score of 4 (severe) on a single HTI item; or at least two injuries which score 3 (moderate) or higher in different organ systems or body regions (loss of blood following an already rated cause is thereby not seen as a separate injury). Our last definition (major emergency treatment) includes patients who urgently need major treatment, which is defined as an emergency thoracotomy, laparotomy, neurosurgery or immediate admission to an intensive care unit or death within $48 \mathrm{~h}$ after the accident.

\section{Data collection}

The Community Health Service in Enschede has established a computerized database. Each action taken by ambulance personnel, data on patients, their condition and the therapy administered are recorded by the emergency medical care assistant. Information is available on the components of the RTS (blood pressure, respiratory rate and the components of the Glasgow Coma Scale), personal characteristics (e.g. name, date of birth and sex), selected characteristics of the accident, the therapy given and information on the time and duration of the emergency assistance. All the information on the study population in this database was reviewed retrospectively by the researcher.

In addition to the prehospital data of the Community Health Service, this study used clinical data from the two hospitals in the region. The hospital records of the patients included were reviewed by the researcher. When a patient was admitted to a hospital, the hospital discharge letter was used for abstracting the medical diagnosis. When only the emergency room was visited, the treatment sheet was reviewed. All patients had been physically examined by the surgeon on duty. After routine examination, specific diagnostic procedures had been undertaken when indicated. Based on the clinical data, the HTI-ISS could be constructed as a 'severity index'. The 'administration of an emergency treatment' and 'survival' were also registered, which together could be used as an alternative severity index. The collection of the 'hospital data' and, subsequently, the scoring of the severity according to the HTI-ISS were done manually by the researcher, under supervision of a surgeon.

For 330 patients ( 83 per cent) both the prehospital and hospital data were complete. Of the 343 patients with an RTS equal to 12 , details for 18 per cent could not be completed ( 37 cases because of errors in the dataset of the Community Health Service, 18 because of missing information in the hospital and six cases for both reasons). Of the 55 patients with an RTS $<12,13$ per cent could not be completed (for five patients no data could be found in the hospital, for one the data in the ambulance department were incomplete and for one both applied; the initial RTS value of all incomplete patients was 11). 


\section{Data analysis}

The study population consists of two classes, patients with an RTS of 12 and all patients with a lower RTS. In both classes the number of patients with major injury according to the different definitions is known. To estimate the prevalence of major injuries in the original population, the patients in the class with the normal RTS were weighted with the reciprocal value of the sample fraction (657/ $343=1.92$ ). To indicate the relationship between the RTS and the existence of major injury, the following parameters were estimated: (i) sensitivity, the percentage of major injuries diagnosed by a lowered RTS; (ii) specificity, the percentage of minor injuries with a maximum RTS; (iii) the predictive value of a positive test $(\mathrm{PV}+)$, which is the percentage of patients with major injury among patients with a lowered RTS; and (iv) the predictive value of a negative test (PV - ), which is the percentage of minor injuries among the patients with a maximum RTS. These parameters offer an impression of the performance of the RTS as a triage instrument in the prehospital setting.

The predictive value can be calculated from the number of patients with major injuries in the two classes. To estimate the sensitivity and specificity, the patients in the sample with a maximum RTS are weighted with the reciprocal value of the sample fraction. In all analyses the initially measured RTS has been used. Two different forms of modification have been analysed. First of all, the RTS is corrected for temporary loss of consciousness. When a lowered RTS was only caused by the Glasgow Coma Scale (GCS) and the GCS normalized during transport, the normal GCS was used to calculate the RTS. Secondly, with a logistic regression analysis the influence of age, sex and type of accident on the relationship between RTS and major injury was analysed. First a model was fitted with severe injuries as the dependent variable and the RTS equal to 12 versus less than 12 as the independent variable. This model was used to analyse whether the modifying variables created an additional risk of diagnosing severe injuries.

\section{Results}

The main characteristics of patients with a normal and a lowered RTS are given in Table I. Of all patients, 73 per cent were victims of a road traffic accident; bicycle accidents form an especially large proportion of all accidents in our study population. The contribution of violence is relatively small. Both classes are more or less similar with regard to the sex and age of the patients, the type of accident, the time needed for the ambulance to arrive for the victim and the time to transport the patient to the hospital. Patients with a lowered RTS needed a longer preclinical treatment time and had a higher mean HTI-ISS score.

An estimate of the prevalence of major injuries in the original population of accident victims is given in Table II. The prevalence of trauma centre candidates in the population under study is low, regardless of the definition used. The highest prevalence was found for the least stringent definition: almost 6 per cent of the accident victims had an HTI-ISS of 18 or over.

The estimated number of patients with major injury among the patients with different RTS scores is given in Table III. Although the chance of severe injuries increases with the lowering of the RTS, a substantial proportion of
Table I. Main characteristics of the study population

\begin{tabular}{|c|c|c|}
\hline & $\begin{array}{l}R T S=12 \\
(N=282)\end{array}$ & $\begin{array}{l}R T S<12 \\
(N=48)\end{array}$ \\
\hline \multicolumn{3}{|l|}{ Age group } \\
\hline $15-29$ & $45.7 \%$ & $41.7 \%$ \\
\hline $30-64$ & $37.6 \%$ & $41.7 \%$ \\
\hline Over 65 & $16.7 \%$ & $16.7 \%$ \\
\hline Mean age (years) & 39.0 & 39.2 \\
\hline So & 20.3 & 20.2 \\
\hline \multicolumn{3}{|l|}{ Sex } \\
\hline Male & $59.4 \%$ & $70.2 \%$ \\
\hline Female & $40.6 \%$ & $29.8 \%$ \\
\hline \multirow{2}{*}{\multicolumn{3}{|c|}{$\begin{array}{l}\text { Type of accident } \\
\text { Traffic }\end{array}$}} \\
\hline & & \\
\hline Automobile & 25.9 & 27.1 \\
\hline Motorcycle & 19.1 & 8.3 \\
\hline Bicycle & 26.6 & 35.4 \\
\hline Pedestrian & 1.8 & 4.2 \\
\hline Falls & 11.0 & 10.4 \\
\hline Violence & 1.4 & 2.1 \\
\hline Other & 14.2 & 12.5 \\
\hline Mean time to arrive at the patient & $6.2 \mathrm{~min}$ & $6.4 \mathrm{~min}$ \\
\hline Mean time for prehospital treatment & $8.6 \mathrm{~min}$ & $13.9 \mathrm{~min}$ \\
\hline Mean time for transport to hospital & $8.0 \mathrm{~min}$ & $9.8 \mathrm{~min}$ \\
\hline \multicolumn{3}{|l|}{ Injury Severity score } \\
\hline $0-8$ & 84.0 & 58.3 \\
\hline $9-17$ & 12.1 & 14.6 \\
\hline $18-24$ & 2.1 & 2.1 \\
\hline 25 or more & 1.8 & 25.0 \\
\hline
\end{tabular}

Table II. Estimated prevalence of serious injury among all accident victims aged over 15 who were alive at the time the ambulance arrived (patients with a normal RTS are weighted with the reciprocal value of the sample-fraction)

\begin{tabular}{lc}
\hline Definition & $\%$ \\
\hline HTI-ISS of 18 or more & 5.8 \\
HTI-ISS of 20 or more & 3.7 \\
Modified HTI-ISS criterion $^{*}$ & 5.3 \\
Major emergency therapy $^{\dagger}$ & 2.7 \\
\hline
\end{tabular}

"Patients with at least one score of 4 in one HTI region or at least two scores of 3 in separate organ regions (blood loss following an already rated cause is excluded).

${ }^{\dagger}$ Emergency thoracotomy, laparotomy, neurosurgery or immediate admission to an intensive care unit or death within $48 \mathrm{~h}$.

Table III. Estimated number of seriously injured patients according to different definitions (patients with a normal RTS are weighted with the reciprocal value of the sample fraction)

\begin{tabular}{lrrrr}
\hline & \multicolumn{5}{c}{ RTS score } \\
\cline { 2 - 5 } Definition & 12 & 11 & 10 & $<10$ \\
\hline HTI-ISS $\geqslant 18$ & 21 & 4 & 3 & 6 \\
HTI-ISS $\geqslant 20$ & 10 & 4 & 3 & 5 \\
Modified HTI-ISS criterion ${ }^{*}$ & 17 & 4 & 4 & 6 \\
Major emergency therapy $^{\dagger}$ & 4 & 4 & 3 & 5 \\
Total number $^{*}$ & 540 & 26 & 13 & 9 \\
\hline
\end{tabular}

"Patients with at least one score of 4 in one HTI region or at least two scores of 3 in separate organ regions (blood loss following an already rated cause is excluded).

${ }^{\dagger}$ Emergency thoracotomy, laparotomy, neurosurgery or immediate admission to an intensive care unit or death within $48 \mathrm{~h}$. 
Table IV. Estimates of sensitivity, specificity and predictive value of the RTS (patients with a normal RTS are weighted with the reciprocal value of the sample fraction)

\begin{tabular}{lccc}
\hline Definition & Sensitivity & Specificity & PV+ \\
\hline HTI-ISS $\geqslant 18$ & $38 \%$ & $94 \%$ & $96 \%$ \\
HTI-ISS $\geqslant 20$ & $56 \%$ & $94 \%$ & $98 \%$ \\
Modified HTI-ISS criterion ${ }^{*}$ & $45 \%$ & $94 \%$ & $96 \%$ \\
Major emergency therapy & $76 \%$ & $94 \%$ & $99 \%$ \\
\hline
\end{tabular}

*Patients with at least one score of 4 in one HTI region or at least two scores of 3 in separate organ regions (blood loss following an already rated cause is excluded).

'Emergency thoracotomy, laparotomy, neurosurgery or immediate admission to an intensive care unit or death within $48 \mathrm{~h}$.

the patients who are trauma centre candidates according to the different definitions have a normal RTS.

The relationship between the RTS and the existence of serious injury is summarized in Table IV. According to three of the four definitions, the sensitivity is lower than 60 per cent. The sensitivity is highest for diagnosing patients who urgently need major treatment, followed by patients who had an HTI-ISS of 20 or over. The predictive value of a positive test is low for all criteria (25-29 per cent), which means that three out of four patients with a lowered RTS do not have major injury and should not be considered as a trauma centre candidate.

To investigate modifying effects, the RTS is first modified in order to rule out the impact of temporary loss of consciousness. This produces a slight improvement in the predictive value and the specificity, without a lowering of the sensitivity. The positive predictive value is 39 per cent (HTI-ISS $\geqslant 18$ ), 33 per cent (HTI-ISS $\geqslant 20$ ), 42 per cent (modified HTI-ISS criterion), and 36 per cent (major emergency therapy). The specificity becomes 96 per cent for all categories and the PV - varies between 97 per cent and 99.7 per cent. The sensitivity remains the same as the values presented in Table $I V$, for all definitions. No modifying effect on the relationship between the RTS value and the existence of serious injury was found for three variables under study: age and sex of the patient and type of accident.

\section{Discussion}

The performance of diagnostic procedures in identifying persons with a disease is in general dependent on two factors: first the qualities of the procedure itself (which is expressed by the sensitivity and specificity) and second the prevalence of the disease in the population. In this study the performance of the RTS as a triage instrument was evaluated in a prehospital setting in The Netherlands. From the results it seems that the prevalence of severe injuries in this setting is low. Of the diagnostic qualities, the sensitivity seems to be the most relevant measure. The sensitivity in our results seems to be lower than in other studies. When severe injuries are defined by the HTI-ISS, 40 to 60 per cent of all trauma centre candidates are identified by the RTS. From our results it appears that the specificity of the RTS is not a great problem: more than 90 per cent of the slightly injured patients were identified that way by the RTS. As a result of the low prevalence of major trauma in the prehospital setting and the low sensitivity of the procedures, the chance that a patient with a lowered RTS does have severe injuries $(\mathrm{PV}+$ ) is only 25 per cent. $A$ slight improvement in the positive predictive value was found after the RTS was corrected for temporary loss of consciousness.

Only the study by Gilpin et al. ${ }^{\circ}$ reported a prevalence in the same range as our study. Several factors can explain the low prevalence of patients with major injury. First, it appears from international comparisons that the incidence of road traffic accidents in The Netherlands is low ${ }^{12,13}$. Since this category accounts for the majority of all accidents in our population, this can explain the low prevalence. Second, most other studies on the performance of the RTS were conducted on an in-patient population, especially among patients who visited a trauma centre. These studies therefore refer to a selected population of more seriously injured patients compared with our study involving accident victims in a prehospital setting. A low prevalence of major injury influences the possibilities of recognizing trauma centre candidates. The performance of the RTS in this study seems to be different from the results of other studies. Compared with the studies of Champion ${ }^{5}$ and Gilpin ${ }^{\circ}$, the sensitivity and the predictive value of a positive test is low and the specificity is high. When the relationship of the RTS with the necessity of urgent therapy is compared with the study of Emerman ${ }^{8}$, all measures lie within the same range.

Several factors can explain the relatively low sensitivity. First, it has to be stressed that patients who were obviously dead at the time the ambulance arrived were excluded. Including this category gives a high sensitivity but this number is less meaningful for the purposes of our study. Second, the numbers in question should be interpreted with caution because the number of patients that were found to have serious injuries is small. The patients with a lowered RTS for which the data could not be completed have probably not introduced any bias in the estimation of the sensitivity. All these patients had a RTS value equal to 11 , and the chance that they had severe injuries is low.

Another explanation for a low sensitivity can be found in the time at which the RTS is measured; it is possible that great loss of blood has not yet resulted in a lower systolic blood pressure.

No comparison could be made between the distribution of the HTI-ISS in the population studied and other studies. This is nevertheless a relevant parameter for the sensitivity and specificity. The sensitivity can be influenced by the distribution of case severity among seriously injured patients. Of the patients with major injury (HTI-ISS of 18 or over) only 40 per cent had an HTI-ISS value of over 24 (which means at least one very severe injury or at least one severe and one moderate injury). This indicates that 60 per 
cent of the patients with major trauma have injuries which make it difficult to distinguish them from patients with minor trauma and results in a lower sensitivity.

The specificity in this study was high for all definitions of serious injuries used. Specificity was especially high after a correction was made for patients with a temporary loss of consciousness. The high specificity can be explained by the distribution of the HTl-ISS among patients who are not seriously injured.

A lowered RTS has a low predictive value in this study. This is mainly the consequence of the low prevalence of serious injury in our population. Three out of every four patients with a lowered RTS was not seriously injured (irrespective of the definition used), and after correction for temporary loss of consciousness the figure is almost two out of every three patients.

This study indicates the limitations of the applicability of the RTS as a triage instrument in the prehospital setting in The Netherlands. These limitations emanate in the first place from the low prevalence of serious trauma. Secondly the situation in which a triage decision has to be taken is relevant. The results of this study refer to a relatively small number of patients in one region of The Netherlands and further research is indicated to confirm these results.

When a triage decision in the prehospital setting is based only on the value of the RTS, in areas with a low prevalence of patients with major injury, there will be the chance of both 'over-triage' and 'under-triage'. Undertriage is an important problem because it limits the potential benefits of a regionalized trauma care system. Over-triage will not only result in inefficient use of resources but will also reduce their effectiveness. Efforts that, in retrospect, prove unnecessary discourage trauma centre and ambulance personnel, in turn reducing the alertness of the system.

Further directives for the triage for accident victims, and for deciding whether or not to transport a patient to a trauma centre, need to be developed. The combination of signs of anatomical injury and the mechanism of injury needs further attention ${ }^{14.15}$. Before such a new instrument is introduced, validation has to take place in the population where it will be applied.

\section{References}

1 West JG, Trunkey DD and Lim RC. Systems of trauma care: a study of two counties. Arch Surg 1979; 114: 455.
2 Draaisma JMTh. Preventable trauma deaths in The Netherlands, a prospective multicenter study. J Trauma 1989; 29: 1552.

3 van Beeck EF, Mackenbach JP, Looman CWN et al. Determinants of traffic accident mortality in The Netherlands: a geographical analysis. Int J Epidemiology 1991; 20: 698.

4 Dutch College on Hospital Facilities. Report on Trauma Care. [College voor ziekenhuisvoorzieningen. Rapport Inzake Traumazorg]. Utrecht: The Netherlands, 1991.

5 Champion HR, Sacco WJ, Copes WS, Gann DS, Gennarelli $\mathrm{TA}$ and Flanagan ME. A revision of the trauma score. J Trauma 1989; 29: 623.

6 Gilpin DA and Nelson PG. Revised trauma score: a triage tool in the accident and emergency department. Injury 1991; 22: 35 .

7 Emerman CL, Shade B and Kubincanek J. Comparative performance of the Baxt trauma triage rule. Am J Emerg Medicine 1992; 10: 294.

8 Emerman CL, Shade B and Kubincanek J. A comparison of EMT judgment and prehospital trauma triage instruments. J Trauma 1991; 31: 1369.

9 Mackenzie EJ, Steinwachs DM and Ramzy AI. Evaluating performance of statewide regionalized systems of trauma care. J Trauma 1990; 30: 681.

10 American College of Surgeons, Committee on Trauma. Field categorization of trauma patients and hospital trauma index. Bull Am Coll Surg 1980; 65: 28.

11 Goris RJA. The injury severity score. World J Surgery 1983; 7: 12 .

12 Lamm R, Chouieri EM and Kloeckner JH. Accidents in the US and Europe: 1970-1980. Accid Anal Prev 1985; 17: 429.

13 van Beeck EF and Mackenbach JP. Accident Monitoring Report 3: Developments since 1985. The Hague, The Netherlands, 1992.

14 Wisner DH. History and current status of trauma scoring systems. Arch Surg 1992; 127: 111.

15 Knopp R, Yanagi A, Kallsen G, Geide A and Doehring L. Mechanism of injury and anatomic injury as criteria for prehospital trauma triage. Ann Emerg Med 1988; 17: 895.

Paper accepted 14 November 1995.

Requests for reprints should be addressed to: J. Roorda, Community Health Service Regio Twente, PO Box 330, 7600 AH Almelo, The Netherlands. 